\title{
Perception of Teaching and Learning Using Medical Simulation Methods Related to Primary Psychological Counseling and Prevention-a Multicultural Focus Group Study
}

\author{
Aleksandra Rutkowska ${ }^{1}$ (D) \\ Published online: 3 January 2018 \\ (C) The Author(s) 2018. This article is an open access publication
}

\begin{abstract}
Medical simulation, especially role-playing, allows students to learn how to practice medicine and help patients without the risk of harming them. A subject, which behaviorally helps to prepare medical students for the role of the doctor is Medical Psychology. Psychology deals with behavior-human functioning in different situations and under an influence of various psychological factors. The aim of the study was to describe how first and second year medical students learn and perceive their role-playing in simulated methods used to improve communication skills. This study applied a retrospective qualitative analysis of interview performance featuring feedback from first and second year medical students and judges. In each scenario, medical students were required to provide clinical consultation to patient-actors in accordance with their knowledge of Behavioral Sciences and Applied Medical Psychology. After the exercise, all participants were instructed to write down their opinions upon their experiences. Particularly important were the self-evaluation reports regarding the emotional state and body language. The reports were contrasted with the impressions of two observing judges who are psychologists. The results reflect conclusions drawn from the thematic analyses as well as from the written remarks of self-reflection regarding the students' performance from two independent evaluators. All of the students who took part in the scenarios that were assuming the role of a doctor reported feeling a lack of psychological competence used in communication. Most of the participants noted difficulty in delivering bad news to aggressive or demanding patients, along with cancer patients. Students over the age of 30 were more likely to handle these situations appropriately. This study offers an insight into how students studying in Poland experience challenges while acquiring proper communication skills. Notably, older medical students dealt with simulated patients better. However, these skills can be taught especially to younger medical students through dedicated training. All results and discussions allow to recommend an unambiguous simulation method suitable to the needs of future doctors in training.
\end{abstract}

Keywords Multiculturalism $\cdot$ Medical simulation $\cdot$ Role-playing $\cdot$ Psychology $\cdot$ OSCE

\section{Introduction}

During the first and second year of studying, medical school students focus their attention mainly on exploring anatomy and physiology, along with other subjects regarding the detailed structure and biochemistry of human functioning. An

Aleksandra Rutkowska

alexarutkowska@gmail.com; http://www.csmlublin.pl/index.php/ en/

1 Department of Didactics and Medical Simulation, I Faculty of Medicine, Medical University of Lublin, Chodzki 19 Street, 2nd Floor, 20-400 Lublin, Poland additional subject, which helps to prepare medical students for the role of the doctor, is Medical Psychology. Especially important for medical students is the psychology of those who are sick, suffering, or mentally disturbed. Psychology deals with behavior-human functioning in different situations and under an influence of various psychological factors [1]. These factors also affect the patient's motivation to cooperate with their doctor. Various studies have shown the attitude of the doctor and the skills of empathetic listening and communication to influence the success of treatment [2]. Therefore, psychological skills have an important role in the education of medical students. The primary communication skills include making contact with the patient, conducting an interview in conditions of limited privacy, and transferring information. In 
addition to different techniques of verbal and nonverbal communication, it is important for students to understand the meaning of empathy and to know how to listen actively [3-5]. Psychology classes, which are conducted in most medical schools, enable students to assume a role of the messenger while being monitored and supported by the teacher. The main goal of study and activities presented within was to assess and improve the students' skill development while at the same time allowing them to plan and think in safe conditions how best to manage the patients' care using their psychological resources. Additionally, the aim was to investigate the judges' and the students' perception of simulation-based methods related to primary psychological counseling and prevention. The simulation methods, although common in teaching Medical Aids or Basic Clinical Skills at the Medical University of Lublin, are being introduced for the first time in regard to teaching psychological skills to students of Medical Psychology. Every student was asked to role-play as a doctor and a patient in a particular arranged scenario, concerned with psychological consultations and prevention. Situations presented in the scenarios required from them to provide help to people in various health emergencies, critically ill in relation to mental and physical health along with those who were posing a threat to the physician, such as those who are aggressive, drug addicted, or mentally impaired patients. In every scenario, despite the established framework, the process happened spontaneously, according to the state of mind and actions of an individual student (actor). After each scenario, performance of the doctor-student and the patient-student was analyzed, and students were asked for feedback based on the assigned role and their own feelings.

\section{Materials and Methods}

The study applied a retrospective qualitative analysis of performance with which first and second year students were able to conduct an interview. Participants (students) were asked to take part in simulated clinical scenarios, instructed to assume the role of the physician or the patient, or a family member. The students were required to provide clinical consultation in accordance with knowledge of behavioral sciences and applied medical psychology. Students who played the roles of patients and family members were abided by the same rules as physicians, but their role was to use their understanding of medicine to rationalize why they should not receive treatment and to question the doctor.

The main content of each simulated session was not known to the students. Their performance lasted for maximally $15 \mathrm{~min}$, and the sessions were video-recorded upon permission for qualitative analysis. After each scenario, each team was briefed on their performance, thoughts, and feelings related to their assigned case. Remarks featuring feedback were recorded for all participants. Qualitative analysis included understanding of student performance in the dynamically simulated scenarios. Each team was culturally diverse and scenarios included a variety of different sociocultural situations. After the exercise, students were prompted to write down brief notes about their experiences and feelings regarding their performance. Particularly important were the notes based on the self-evaluation of expressed emotions and the body language used. The notes were later juxtaposed with the impressions reported by two observing judges (two trained psychologists). Their observations allowed us to evaluate student's performance in a given dynamic scenario and to contrast their selfperceived evaluation with expert judges. The analyses allowed us to consider how such factors as pride and leadership skills influenced decision making and self-perception from the perspective of an outside psychological observation. Thanks to the recorded scenarios, we were able to understand the students' learning process along with their perception of themselves and their personal self-evaluations.

For the analysis, the documentation of 7 simulation acts, involving 15 first year students of medicine was used. Among the participants there were 10 men and 5 women. The students ranged in age from 21 to 28 years old and came from the USA, Poland, and Taiwan. Ethnically, three participants were Black (from the USA), one Asian (from Taiwan), and the rest were Caucasians (a mix of students coming from the USA and Europe). Simulation scenes were a part of a practical subject, "Medical Psychology." Every participant was instructed separately regarding their role and the setting in which they had to play in the scenario. The further course of the exercise was spontaneous. After the end of the simulation, the students were asked to perform self-assessment while judges provided their own observations. Collected data were used in further analysis.

Data analysis was performed using a method of a qualitative analysis, along with the principles of grounded theory. Firstly, all written reports and remarks were collected into a single database and assessed using MaxQDA, version 12. After the database was created, two researchers independently coded text lines with unique codes, which represented a contextual representation of the main theme of the discussion line. Subsequently, the codes were combined thematically to yield main topic points. Reported results reflect conclusions drawn from the thematic analyses as well as from the remarks on selfreflection of the students' performance and evaluation from two independent judges.

\section{Results}

The results suggested that all students which took part in the scenarios, from the role of the doctor, reported feeling that they were lacking practical communication skills in terms of psychological competence. Most of the students demonstrated conversational difficulties when it came to appropriate word 
use, content of speech, or the appropriate form on information used in trauma scenarios.

At that point, an interesting question was posed, mainly: what was responsible for the inability to communicate effectively, a lack of language skills, shyness, over-appreciation of clinical skill over communication ones, or other factors? As evaluated self-reported data shows, students often exhibited narcissistic traits which might have prompted them to falsely believe that basic medical knowledge should be prioritized in the context of medical care. Many described their own performance as highly proficient and informative, which was in disagreement when compared to the reports of the psychologist evaluators. This case appears to be similar to those described by the existing literature that consistently show that medical professionals prioritize medical knowledge over communication skills. The results have further ramifications for the ongoing debate about how medical students should learn to deliver medical knowledge to people.

In addition, the most noted difficulty was caused by an inability to transfer bad news to simulated patients (cases 1, $2,3)$. While students noted that they had difficulty dealing with patients, who became frantically frustrated with their diagnosis, not a single participant offered a clue as to why they failed at delivering the bad news. Psychologist evaluators have found that students started performing poorly in transferring bad information to their stimulated patients as soon as the patient raised their voice, often inviting family members to join the conversation. With more people present in the room, the participants typically became confused as to who they were talking to regarding the diagnosis.

Students reported fear and anxiety associated with scenarios that required them to perform consultations at the patient's home. The main reason stated by the participants was an inability to feel comfortable while being in an unfamiliar place, e.g., previously mentioned house in which the patient lives.

In case of contact with an aggressive or demanding patient, or providing information about the irreversible damage to the body, students struggled tremendously. All of the participants did not know how to handle the situation as soon as two or more students acting as the patient and family members expressed resentment and disapproval for their advice. Questioning the doctor's training almost always instantly constricted the doctor's ability to continue the conversation with the patient. Questions about the quality of medical education were particularly hurtful. Students reported in their remarks that as soon as the patient questioned their authority and knowledge, they felt violated and unjustifiably attacked. There was no evidence of students reflecting on the principle of not taking patients, who were questioning their level of competence serious. Therefore, basic clinical preparation through behavioral science training may be insufficient to prepare students that are going to face disrespectful patients.

Difficulties in understanding the needs of a given patient accompanied the students especially in situations of confrontation with patients from different races or cultures, who may express emotions differently (cases 2, 5, 6). Also, it is important to note that there was difficulty involved in terms of conducting a full medical history review and providing a prognosis for treatment of children and the mentally ill patients (cases 4 and 5).

Maturity mattered as well - the older the student was, the more conscientious he or she was about acting the role of the physician. What was worth noting, they also listened attentively along with collecting notes throughout the interviews whereas their junior counterparts appeared to be less prone apt at these skills.

Interestingly, Black students reported feeling the obligation to play an assertive doctor. They claimed that the cultural perceptions towards Black people influenced their tendency towards louder verbalizations and more unrestricted body postures during the scenarios. Another interesting finding was that the students from Asian countries actually reported a difficulty with expressing their emotions. These students noted that they never thought that the doctor should be good with expressing feelings since body language does not affect the efficiency of a good doctor; however, they did acknowledge that good clinical judgment does.

Feedback from all of the participants indicates that cultural background and educational background largely influenced their ability to show openness, good communication skills, and signify respect for traditional expressions of emotions. Without a doubt, mastery of the language matters too; some students did better with choosing appropriate words than others, which reflected their educational background and influenced execution of the task.

Contact with the patients from different ethnicities was perceived as difficult, even though the students recognized that the simulated patients were their classmates playing the roles. Intrinsic racial beliefs inevitably influence perceptions about others, not only in real-life situations, but also in simulated scenarios. Discouragement expressed by patients during conversations generally resulted in negative emotions and aroused a misunderstanding between the physician and the patient.

Most of the students reported feeling tired after partaking in two scenarios. Furthermore, all participants expressed frustration over their colleagues which were difficult to cooperate with. Not always, however, students were able to learn from their experiences gained during the scenarios, which was confirmed by the self-reflection which was confirmed by the selfreflection provided by the participants written remarks (Table 1).

\section{Discussion}

Specialists of medical education agree that health professionals require a high level of empathy and communication 


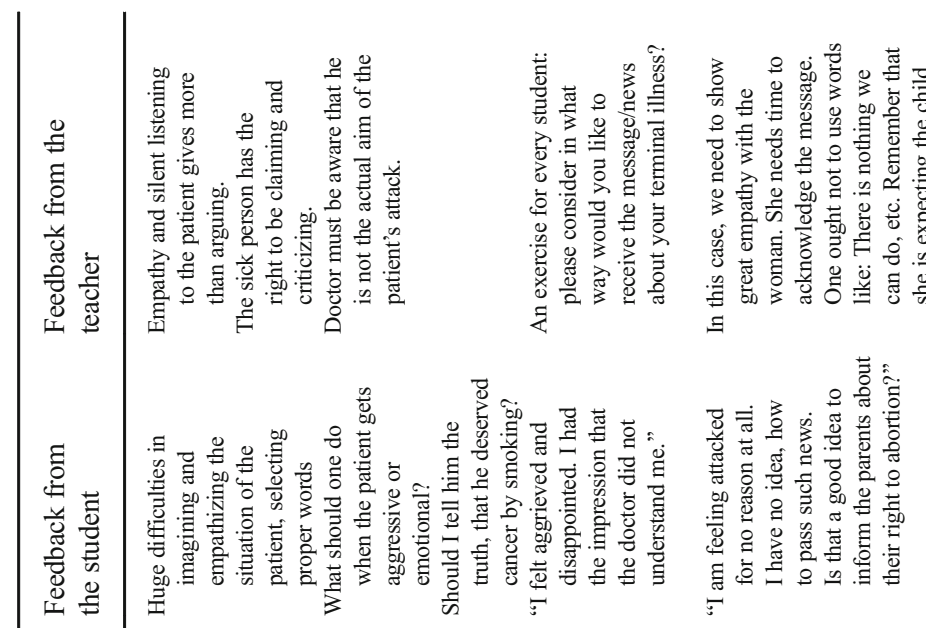

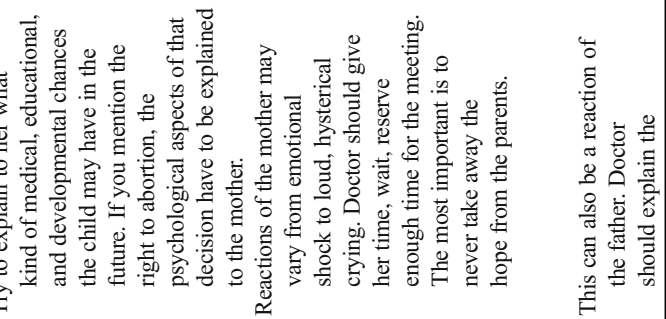

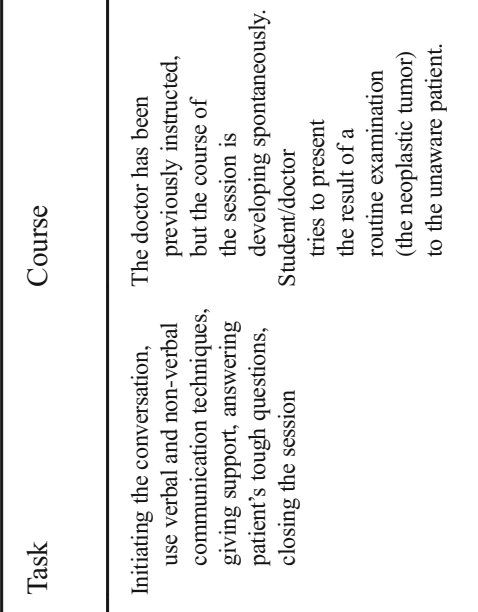

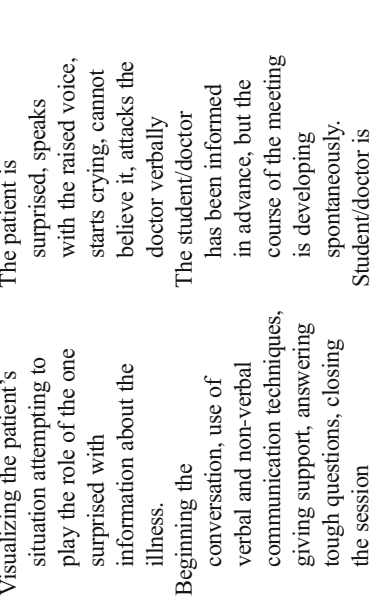

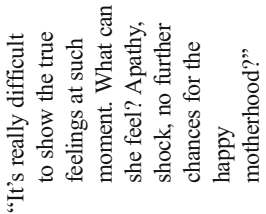

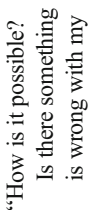

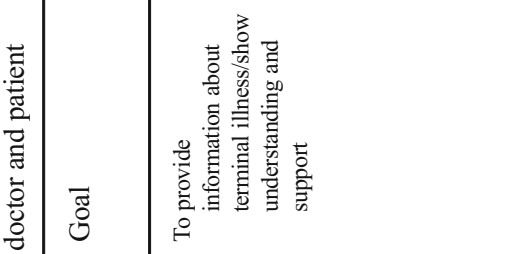

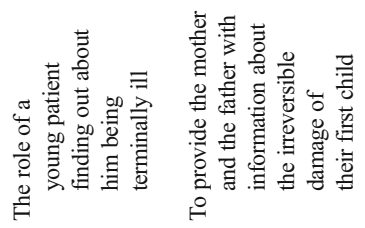

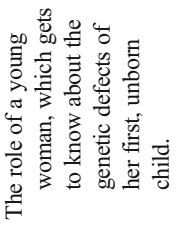

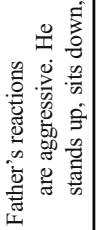

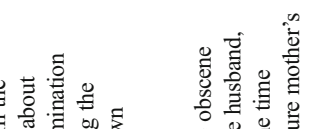

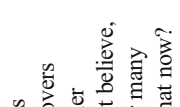

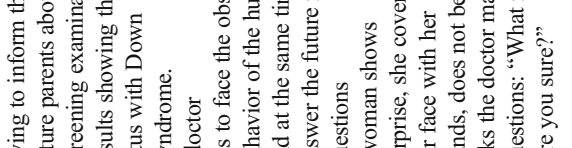

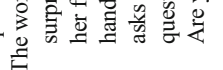
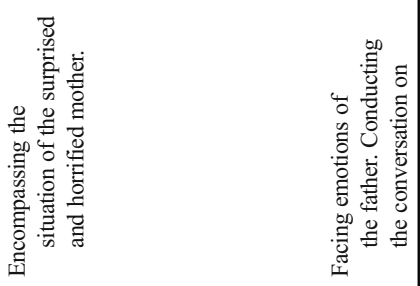

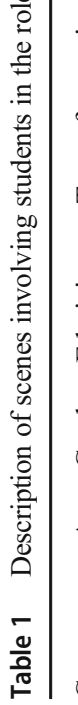

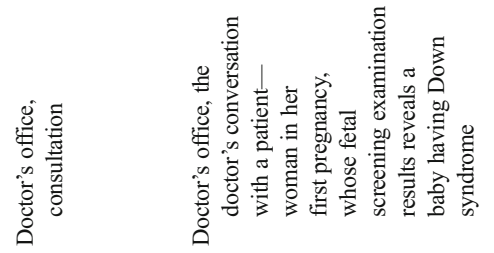

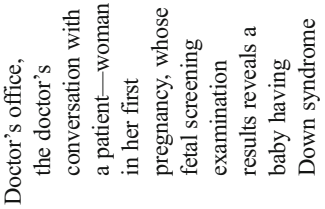

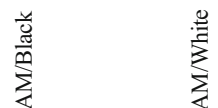

嶨

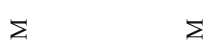

ล

3

2

䓂蒿

离 


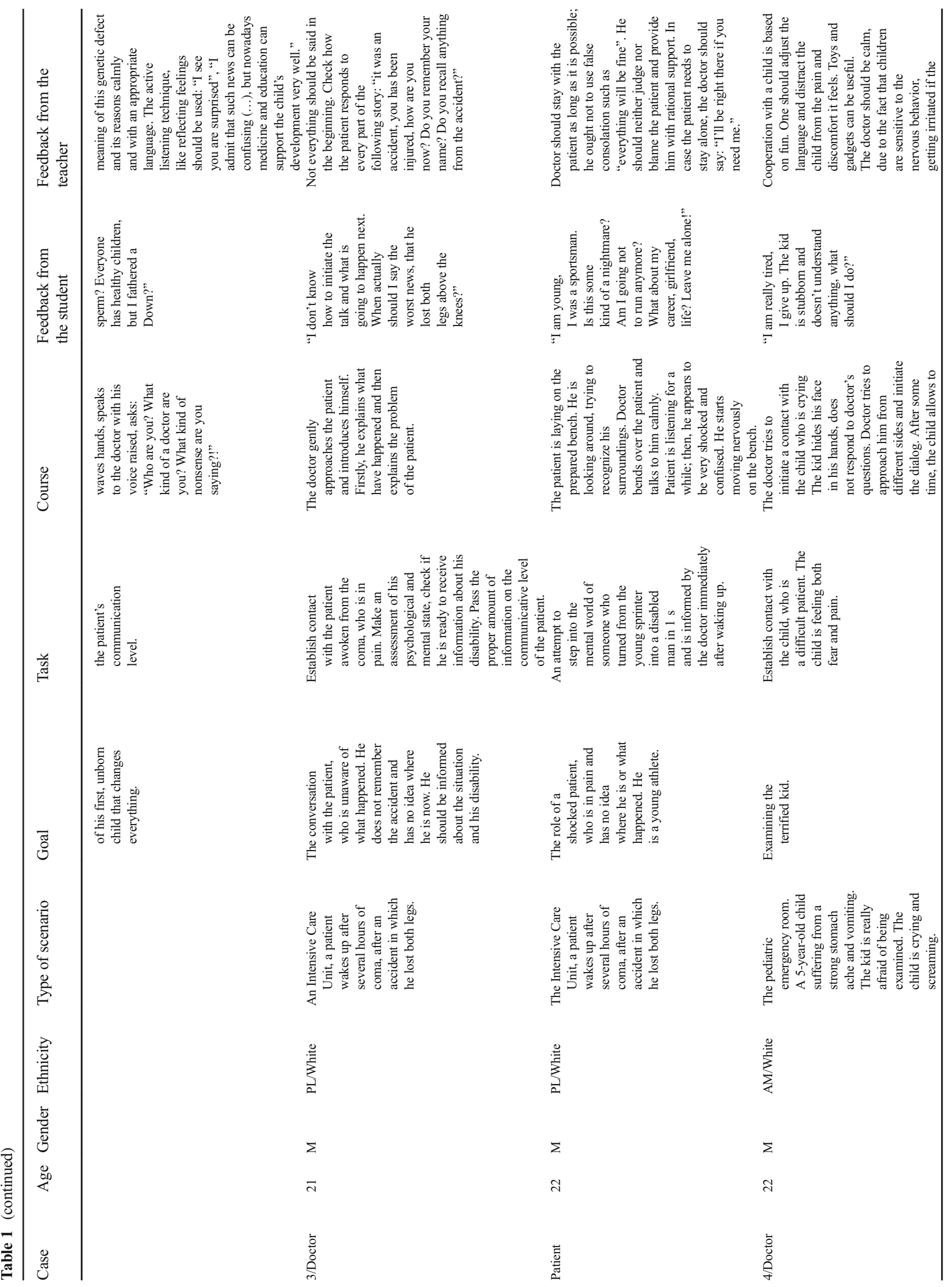




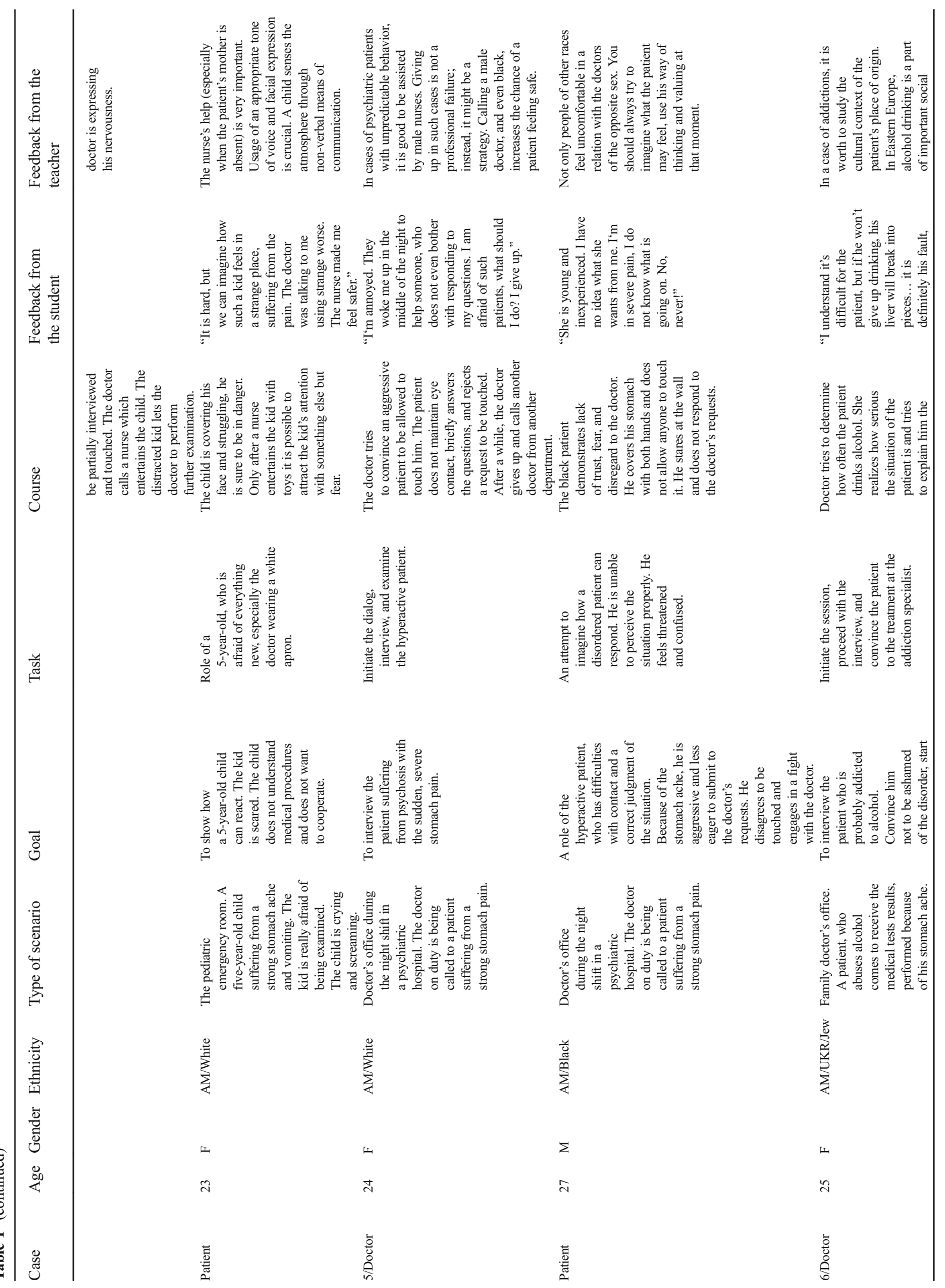




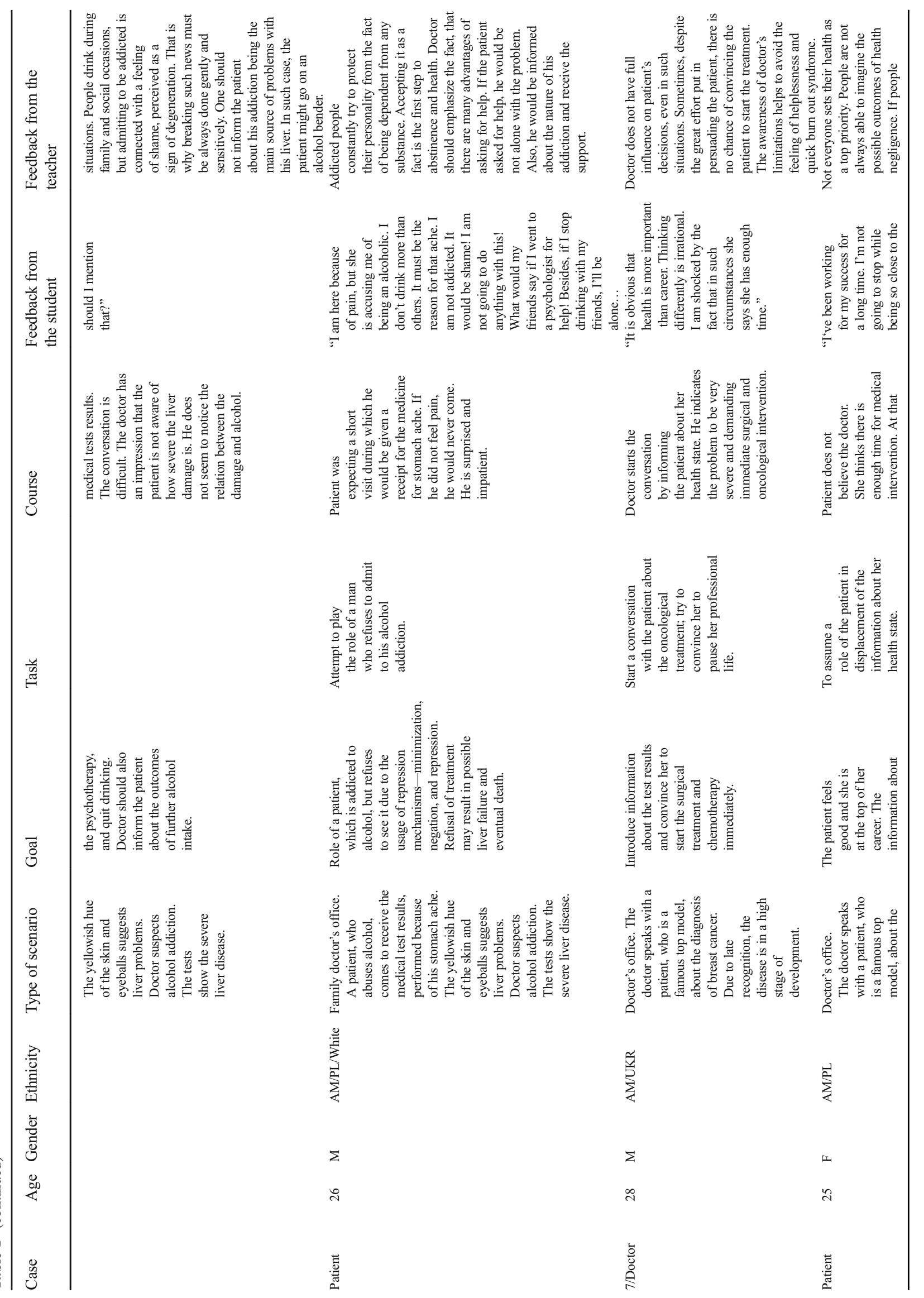


skills in order to be efficient in relation to future patients [6-9]. How can we improve the communication skills training program? The simulation methods can be as effective as traditional contact with a live patient, at the beginning of the medical course in particular [10]. Medical students must be aware of their limitations, especially when it comes to communication and psychological coping. Scenarios which students participated in and reflected on in the form of written remarks indicated that their biggest fear is the inability to distance themselves from their professional duties. Role-playing verifies the sense of self and humility of being a future doctor [11, 12]. Not every patient needs a doctor that has an authoritative demeanor. Instead, however, they may prefer cooperative style, which helps in treating difficult cases.

If doctors conducting clinical classes are not able to prepare young physicians as competent practitioners, who will know how to maturely use their emotions and communication skills to understand the patient? For students having problems with communication, it may be necessary to perform an assessment with psychological tests on communication and personality to individually tailor the simulation-based practice scenarios in order to improve their performance. In addition, one should strongly emphasize the necessity of classroom simulated "doctor-patient" communication being conducted by a psychologist earlier than any clinical classes with a live patient [13].

Following the comments of judges, we see that students should be also taught more about verbal and nonverbal communication. The conclusion is that less important is the content of what you say, but instead how you say it [14]. Medical students should be trained on how to make verbal and nonverbal communication more consistent. Six main emotions that are indicated through mimic expressions in a similar manner are anger, sadness, surprise, happiness, disgust, and fear. The most reliable are the eyes, for their expressions are physiologically dependent and are not subject to conscious control of man. Subconsciously, we realize that the eyes usually reveal our true intentions. Therefore, one of the main symptoms of lying is avoiding eye contact. Another facial element, which is often manipulated, is the mouth. Facial expressions are ambiguous, e.g., a smile can mean joy, embarrassment, ridicule, or contempt. Patients are attentively watching the doctor's face during an interview and waiting for facial expressions even more than for verbal communication, especially during announcing bad news $[15,16]$. A good doctor should also know how to interpret human emotions. Among the most serious mistakes made by the untrained in the study of body language is to interpret individual gestures in isolation from other gestures or circumstances. Listening about the patient's illness (and remembering about the fact that their history is very subjective), doctors should follow their narration entirely-including verbal and non-verbal communication $[15,16]$. There is a variety of communication skills that 


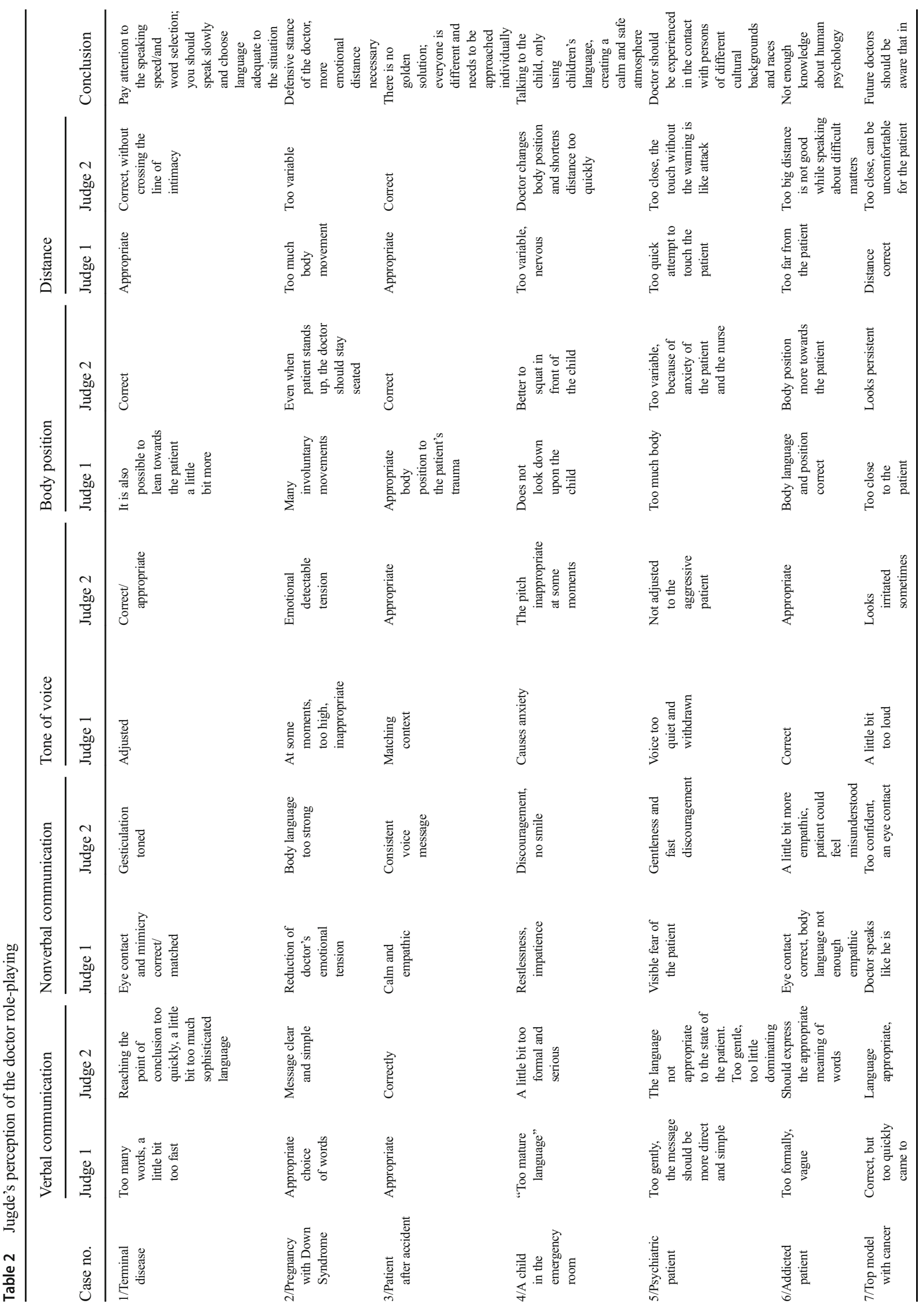


students of medicine should be taught before practicing on live patients.

Feedback allows to take a closer look into the personal resources and limitations of a student in the role of the doctor. This opens up a possibility of deep reflection and letting the student ask themselves questions such as: "Am I strong enough mentally to perform the role of a doctor?", "Am I able to handle the stress accompanying the profession?", and "What are the psychological characteristics I should cultivate?". Another advantage of this method is that it gives the possibility of cooperation between students from different countries as well as belonging to different religions. Thanks to the multicultural student community, they have the possibility of direct contact with students from completely different culture. This allows future doctors to learn how to understand religious and cultural differences and overcome their own prejudices and stereotypes, which should be eradicated from the medical profession. In addition, reacting to various social phenomena, such as violence against women and addiction among people from different cultural backgrounds, opens up opportunities to discuss these issues internationally and compare the prevalence of pathology in various parts of the world. In the study, seven cases of students playing the role of physicians in difficult situations were psycho-medically analyzed and assessed in detail. The remarks and discussions allow for an unambiguous recommendation of a simulation method in the psychological training of future doctors-practitioners. The medical simulation and role-playing method offers a lot of possibilities to be used within medical school (Table 2).

\section{Strengths and Limitations}

The strength of the study is that it contains a comparison of self-reported student opinions about their performances during specific scenarios, while at the same time being compared to the opinions of two experienced psychologists. This analytical approach has allowed me to show how students often overestimate their own competence, although the expert's assessment clearly shows the shortcomings in their preparation to work with patients, and above all, communication problems.

Grounded theory was used in the analysis of the psychological components allowing for the examination of the external aspects that affect the quality of communication between the medical student and the patient. Most of the research in the field of medical communication uses questionnaires to study how doctors and medical students communicate with their patients along with how to convey information. Unfortunately, this method of data collection did not present a complete picture of what factors affect the quality of communication. This study revealed certain elements such as that of, the type of patient (aggressive vs. silent), the setting 
situation (ordinary consultation vs. breaking bad news about cancer diagnosis), as well as other factors such as body language all affect the quality of the conducted consultation. In addition, this study is grounded in the understanding that the student after completing courses in psychology is actually able to reliably reflect what is learned in theory on their medical practice in the hospital.

The main limitation of this study is that it includes only a description of the experiences of one institution of learning; therefore, extrapolation of the results to the global trends is limited. Nevertheless, these experiences may help other institutions that are planning to teach applied psychology through the role-playing method.

The group of respondents is relatively small. Qualitative study, however, generally uses a group of people not counting more than 15 people. The research was based on a group of foreign students studying in Poland; therefore, we cannot create a generalization applicable to students internationally - the research group can be perceived only as a representative of students which visit our university. In order to be able to extrapolate the results, it might be required to conduct an evaluation of a bigger group of participants. Another problem may be that the research has been done on a group of students in a simulated situation where some students played the role of patients and other ones the role of doctors-its results may not fully reflect their behavior in a situation when a similar case occurs in real life. In future studies, an interesting solution would be to watch the students at work with the actual, hospitalized patients, although aspects such as ensuring patient privacy can prevent this type of testing in a clinical setting.

\section{Conclusions}

The possibility to practice psychological competencies in safe, simulated conditions enables students to better prepare themselves to enter the medical profession as practicing physicians. Diversity between ethnic groups gives students an opportunity to widen their knowledge and understanding of how diverse their patients can be in the future. Exercises in psychological skills through simulated scenarios allow future doctors to gain a better understanding of their own emotional reactions, resources, and psychological limitations when confronted with a difficult patient. What is more, the impact of our teachers and judges is very significant. The analysis of verbal, non-verbal communication, body position, and distance was very detailed and significantly contributed to the research. Thanks to the research performed, especially students' feedback, it is now clear that students need more practical exercises in their curriculum to enhance their empathy and psychological skills for their future profession. They also require more psychological training within the medical school to help them better understand their psychological resources and limitations in cases of difficult or aggressive patients.

The manuscript presents the study and the reflections about the way of teaching medical psychology from the sides of both participants and judges. New methods are going to be developed and used to create the standards used to evaluate the communication skills of medical students, to include OSCE, that would further be used to assess the students' level of communication and psychological skills. The Medical University of Lublin is working on introducing OSCE as a permanent way of assessing the students' capabilities of conducting a proper doctor-patient interview.

Acknowledgements I would like to thank my colleague Damian Jacob Sendler, who provided the insight and expertise that greatly assisted in the research. I would also thank the students of II Faculty of Medicine who agreed to participate, and whose feedback enriched my understanding of simulation-based learning.

\section{Compliance with Ethical Standards}

Conflict of Interest The author declares that she has no conflict of interest.

Statement on Ethics This is a retrospective observational study and there are no ethical concerns to declare.

Consent All students participating in the study have voluntarily consented to participate in the scenarios and agreed to have the notes from observational sessions used in future reporting.

Open Access This article is distributed under the terms of the Creative Commons Attribution 4.0 International License (http:// creativecommons.org/licenses/by/4.0/), which permits unrestricted use, distribution, and reproduction in any medium, provided you give appropriate credit to the original author(s) and the source, provide a link to the Creative Commons license, and indicate if changes were made.

\section{References}

1. Bandura A. Toward a psychology of human agency. Perspect Psychol Sci. 2006;1(2):164-80. https://doi.org/10.1111/j.17456916.2006.00011.x.

2. Street RL Jr, Makoul G, Arora NK, Epstein RM. How does communication heal? Pathways linking clinician-patient communication to health outcomes. Patient Educ Couns. 2009;74(3):295-301. https://doi.org/10.1016/j.pec.2008.11.015.

3. Epstein RM, Street RL Jr. Patient-centered communication in cancer care: promoting healing and reducing suffering. 2007. https:// doi.org/10.1037/e481972008-001.

4. Zachariae R, Pedersen CG, Jensen AB, Ehrnrooth E, Rossen PB, Maase von der H. Association of perceived physician communication style with patient satisfaction, distress, cancer-related self-efficacy, and perceived control over the disease. Br J Cancer. 2003;88(5):658-65. https://doi.org/10.1038/sj.bjc.6600798. 
5. Stevenson FA, Barry CA, Britten N, Barber N, Bradley CP. Doctorpatient communication about drugs: the evidence for shared decision making. Soc Sci Med. 2000;50(6):829-40. https://doi.org/10. 1016/S0277-9536(99)00376-7.

6. Quail M, Brundage SB, Spitalnick J, Allen JP, Beiby J. Student self - reported communication skills, knowledge and confidence across standardised patient, virtual and traditional clinical learning environments. BMC Med Educ. 2016;16(1):73. https://doi.org/10.1186/ s12909-016-0577-5.

7. Forrest K, McKimm J, Edgar S. Essential simulation in clinical education. Chichester: Willey-Blackwell; 2013. https://doi.org/10. 1002/9781118748039.

8. Austin EJ, Evans P, Magnus B, O'Hanlon K. A preliminary study of empathy, emotional intelligence and examination performance in MBChB students. Med Educ. 2007;41(7):684-9. https://doi.org/10. 1111/j.1365-2923.2007.02795.x.

9. Hojat M, Vergare M, Maxwell K, Brainard G, Herrine S, Isenberg $\mathrm{G}$, et al. The Devil is in the third year: a longitudinal study of erosion of empathy in medical school. Acad Med. 2009;84(9): 1182-91.

10. Brooks KD, Acton RD, Hemesath K, Schmitz CC. Surgical skills acquisition: performance of students trained in a rural longitudinal integrated clerkship and those from a traditional block clerkship on a standardized examination using simulated patients. J Surg Educ. 2014;71(2):246-53.

11. Davis CM. What is empathy, and can empathy be taught? Phys Ther. 1990;70(11):707-11. discussion 712-5

12. Fallowfield L, Jenkins V. Effective communication skills are the key to good cancer care. Eur J Cancer. 1999;35(11):1592-7. https://doi.org/10.1016/S0959-8049(99)00212-9.

13. Shea SC, Barney C. Teaching clinical interviewing skills using roleplaying: conveying empathy to performing a suicide assessment: a primer for individual role-playing and scripted group role-playing. Psychiatr Clin North Am. 2015;38(1):147-83. https://doi.org/10. 1016/j.psc.2014.10.001.

14. Silverman J, Kurtz S, Draper J. Skills for communicating with patients. London-New York: Radcliffe Publishing; 2005.

15. Andersen PA. Nonverbal immediacy in interpersonal communication. Multichannel integrations of nonverbal behavior. 1985; 1-36.

16. Argyle M. Non-verbal communication in human social interaction. In: Hinde RA, editor. Non-verbal communication. Oxford: Cambridge University Press; 1972. 